\title{
Clinical and economic outcomes after surgical aortic valve replacement in Medicare patients
}

This article was published in the following Dove Press journal:

Risk Management and Healthcare Policy

30 October 2012

Number of times this article has been viewed

\author{
Mary Ann Clark' \\ Francis G Duhay ${ }^{2}$ \\ Ann K Thompson ${ }^{2}$ \\ Michelle J Keyes ${ }^{3}$ \\ Lars G Svensson ${ }^{4}$ \\ Robert O Bonow ${ }^{5}$ \\ Benjamin T Stockwell ${ }^{3}$ \\ David J Cohen ${ }^{6}$ \\ 'The Neocure Group LLC, \\ Washington, DC, ${ }^{2}$ Edwards \\ Lifesciences Corporation, Irvine, CA, \\ ${ }^{3}$ The Burgess Group LLC, Alexandria, \\ VA, ${ }^{4}$ Department of Thoracic and \\ Cardiovascular Surgery, The Cleveland \\ Clinic Foundation, Cleveland, \\ $\mathrm{OH},{ }^{5}$ Center for Cardiovascular \\ Innovation, Department of Medicine, \\ Northwestern University Feinberg \\ School of Medicine, Chicago, IL, \\ ${ }^{6}$ Saint Luke's Mid America Heart \\ Institute, Kansas City, MO, USA
}

Correspondence: Mary Ann Clark The Neocure Group LLC, 1750 K Street NW, 7th Floor, Washington, DC 20006, USA

$\mathrm{Tel}+\mathrm{I} 2025686673$

Fax +l 5083185023

Email clark@neocuregroup.com
Background: Aortic valve replacement (AVR) is the standard of care for patients with severe, symptomatic aortic stenosis who are suitable surgical candidates, benefiting both non-high-risk and high-risk patients. The purpose of this study was to report long-term medical resource use and costs for patients following AVR and validate our assumption that high-risk patients have worse outcomes and are more costly than non-high-risk patients in this population.

Methods: Patients with aortic stenosis who underwent AVR were identified in the 2003 Medicare 5\% Standard Analytic Files and tracked over 5 years to measure clinical outcomes, medical resource use, and costs. An approximation to the logistic EuroSCORE (European System for Cardiac Operative Risk Evaluation) based on administrative data was used to assess surgical risk, with a computed logistic EuroSCORE $>20 \%$ considered high-risk.

Results: We identified 1474 patients with aortic stenosis who underwent AVR, of whom 1222 $(82.9 \%)$ were non-high-risk and $252(17.1 \%)$ were high-risk. Among those who were non-highrisk, the mean age was 73.3 years, 464 (38.2\%) were women, and the mean logistic EuroSCORE was $7 \%$, whereas in those who were high-risk, the mean age was 77.6 years, $134(52.8 \%)$ were women, and the mean logistic EuroSCORE was 37\%. All-cause mortality was $33.2 \%$ for nonhigh-risk and $66.7 \%$ for high-risk patients at 5 years. Over this time period, non-high-risk patients experienced an average of 3.9 inpatient hospitalizations and total costs of $\$ 106,277$ per patient versus 4.7 hospitalizations and total costs of $\$ 144,183$ for high-risk patients.

Conclusion: Among elderly patients undergoing AVR, long-term mortality and costs are substantially greater for high-risk than for non-high-risk individuals. These findings indicate that further research is needed to understand whether newer approaches to aortic valve replacement such as transcatheter AVR may be a lower cost, clinically valuable alternative.

Keywords: aortic valve, replacement, health economics

\section{Introduction}

According to data from the Nationwide Inpatient Sample, the number of aortic valve replacement (AVR) procedures has grown significantly over the years, with approximately 67,500 procedures performed in the US in $2010 .{ }^{1}$ Medicare beneficiaries constitute $63 \%$ of all patients undergoing AVR. ${ }^{1}$ With the aging of the population, the prevalence of aortic valve disease and valve replacement surgery is expected to continue this growth trend. Many published studies have documented the clinical advantages of AVR in patients with severe, symptomatic aortic stenosis. ${ }^{2-13}$ AVR improves clinical outcomes including mortality, reduces symptoms, and improves patient quality of life. Nevertheless, for patients at high risk of surgical mortality, options become increasingly limited, and the potential benefit of AVR must outweigh both the surgical risk 
as well as risk associated with no treatment at all. For this patient population, new, minimally invasive AVR treatments may be their only viable treatment option. ${ }^{14,15}$

Rapidly rising health care costs in the US have caused policymakers to seek increasingly an understanding of the cost-effectiveness of new technologies in order to ensure cost containment while maintaining and improving patient care. High mortality rates coupled with high end-of-life treatment costs for patients with aortic stenosis make this disease an area of particular interest. While AVR remains the standard of care for patients with severe aortic stenosis, few studies have effectively captured the long-term medical care resource utilization and costs for patients following AVR.

Due to the high prevalence of aortic stenosis in older patients, the Medicare population provides a "real-world" representation of the clinical progression of the disease and the associated resource use and costs. Using Medicare claims data, this study seeks to describe the costs and resource use associated with surgical treatment in both non-high risk and high-risk patients.

\section{Materials and methods Data sources and study population}

This study used the Medicare Standard Analytic Files (SAFs), 5\% sample for 2001-2008. While data from 2003 was used to identify the patient population, data from the previous 2 years were used to identify chronic conditions and previous surgeries for risk adjustment, and data out to 2008 were used to identify 5-year costs and outcomes. These files contain all claims (physician, inpatient, outpatient, skilled nursing, home health, hospice, durable medical equipment suppliers, and other suppliers) from a 5\% random sample of Medicare beneficiaries enrolled in the fee-for-service Medicare program. Each patient receives a unique identifier that allows claims to be linked across time for longitudinal analysis of health resource use (services, length of stay), medical costs (Medicare payments), and clinical outcomes (defined by diagnosis and procedure codes, death date). Furthermore, the SAF (denominator) provides information on patient demographics including age, race, and other enrolment-related beneficiary information.

Patients included in this study were first identified by selecting a broader population of both medically managed and surgically treated patients with severe symptomatic aortic stenosis using physician, inpatient, and outpatient claims from 2003 containing an International Classification of Diseases 9th Clinical Modification (ICD9-CM) diagnosis (395.0, 395.2, $396.0,396.2,395.1,395.9$, or 424.1 , or 746.3 ) or procedure code (ICD-9-CM 35.01, 35.96, 35.21, 35.22, or Current Procedural Terminology [CPT] code 92986, 33405) indicating the presence of aortic stenosis. Medically managed patients with severe aortic stenosis were identified based on the presence of a hospital admission with a principal diagnosis of heart failure (ICD-9 DX 398.91, 402.01, 402.11, 402.91, 404.01, 404.11, 404.91, 404.03, 404.13, 404.93, 428.0, 428.1, 428.20, 428.21, 428.22, 428.23, 428.30, 428.31, 428.32, 428.33, $428.40,428.41,428.42,428.9)$ or a balloon valvuloplasty procedure (35.01, 35.96, or CPT 92986) and no surgical AVR procedure. Surgically treated patients with severe, symptomatic aortic stenosis were defined by the presence of a surgical AVR procedure code (ICD9-CM PX 35.21, 35.22, CPT 33405). Continuous enrollment in both Medicare parts $\mathrm{A}$ and $\mathrm{B}$ for the entire follow-up period or until death was also required.

\section{Outcome measures}

Mortality, myocardial infarction rate, and stroke rate were all tracked as clinical outcomes. The SAF (denominator) was used to obtain the date of death. A patient was classified as having had a myocardial infarction if there was at least one hospital admission with a principal diagnosis of myocardial infarction (ICD-9 DX 410.01, 410.11, 410.21, 410.31, 410.41, 410.51, 410.61, 410.71, 410.81, 410.91). Strokes were defined by a hospitalization with a principal diagnosis of stroke (ICD9 DX 430, 431, 434.00, 434.01, 434.10, 434.11, 434.90, 434.91, 435.0, 435.1, 435.3, 435.8, $435.9,436,997.02)$. Additional outcomes that were tracked included rates of repeat AVR, valvuloplasty, admission to a hospice (presence of a hospice claim), admission to a skilled nursing facility (presence of a skilled nursing facility claim), number of acute inpatient hospitalizations (number of acute inpatient claims), hospital days (sum of acute inpatient length of stay), and skilled nursing facility days (sum of skilled nursing facility length of stay).

Costs were measured as payments made by the Medicare program for medical services reported on Medicare parts A and B claims and excluded beneficiary cost sharing, such as coinsurance and deductibles. Costs were computed based on all types of claims and accumulated over the 5-year followup period. Additionally, average follow-up costs per patient per year were calculated by dividing a patient's total follow-up costs by the number of years of follow-up time. Total followup costs exclude costs incurred during the index quarter, which are primarily due to costs associated with the surgical AVR procedure. The robustness of the SAF data allowed us to examine and report on various other components of cost and resource use, which are presented in the tables. These 
components were determined based on Medicare claim type, bill type, and provider number.

\section{Risk stratification and adjustment}

Patients included in the study were classified into highrisk and non-high-risk groups for risk stratification using the logistic EuroSCORE (European System for Cardiac Operative Risk Evaluation, http://www.euroscore.org/ logisticEuroSCORE.htm). Although the risk score calculator developed by the Society for Thoracic Surgery may be more appropriate for use in a US patient population, its application in claims data is not possible because the methodology and formula have not been published. The logistic EuroSCORE predicts surgical mortality for patients undergoing open heart surgery using patient-specific clinical criteria and risk factors including age, gender, comorbid conditions, and previous procedures. We used the SAF (denominator) to obtain the continuous age variable of the patient at the beginning of 2003 to determine age. Each of the other risk factors were determined on the presence of ICD9-CM DX and PX codes on certain claims from the 2 years prior to a patient's index event or index hospitalization. The specific codes used to define each logistic EuroSCORE covariate are provided in Appendix A. All of the risk factors except for age were treated as dummy variables. The values for each patient were included in the logistic EuroSCORE model. High-risk patients were identified by a logistic EuroSCORE $\geq 20 \%$, which has been a definition used to identify high-risk AVR patients in other studies and clinical trials. ${ }^{16}$

Further risk adjustment to account for comorbid conditions not already included in the logistic EuroSCORE model was employed using Medicare's Hierarchical Condition Categories (HCCs). The HCC methodology is used by Medicare to calculate risk-adjusted costs and payments to Medicare Advantage plans, and we used these HCCs to define clinically relevant conditions. ${ }^{17}$ Under this methodology, diagnosis codes on inpatient, outpatient, and physician claims are used to identify comorbid conditions. These comorbid conditions as well as other patient demographic variables are used to calculate an HCC score to adjust monthly Medicare Advantage capitation payments. The risk scores are indexed to a value of 1.0, which represents the costliness of the average Medicare beneficiary. Therefore, a calculated risk score of 2.0 indicates that a patient is twice as costly as the average. In our study, HCC score was only used to report baseline patient characteristics, but we used several clinically relevant HCC groups to adjust for comorbid conditions in the multivariate models.

\section{Statistical analysis}

Continuous variables are expressed as the mean \pm standard deviation and were compared using $t$-tests. Categorical variables were compared using Fisher's exact test and are presented as frequencies and percentages. A Cox regression model was used to predict clinical outcomes. Ninety-five percent confidence intervals and hazard ratios are reported for clinical outcomes. A number of covariates including age, gender, race, region, individual logistic EuroSCORE components, clinically relevant comorbid Medicare HCC conditions, and length of follow-up were used to adjust for baseline differences. Individual logistic EuroSCORE components and $\mathrm{HHC}$ categories were used rather than the actual scores because we wanted to understand the conditions that are most significant in predicting outcomes. All analyses were performed using SAS version 9.2 (SAS, Cary, NC), and a two-sided $P$ value of 0.05 was considered to be statistically significant.

\section{Results}

\section{Patient population}

A total of 3624 patients with severe, symptomatic aortic stenosis (both medically managed and surgically treated) were identified in the 2003 Medicare SAF 5\% sample. From this group, 1474 (41\%) patients were identified as having been surgically treated. The baseline characteristics presented in Table 1 show that their mean age was 74.0 years, $40.7 \%$ were women, $93.1 \%$ were white, and $51.1 \%$ were from the East Region. While the mean logistic EuroSCORE for the group was $12 \%, 17.1 \%$ of the patients had a score $\geq 20 \%$ and were considered high-risk. The mean HCC score for the group was 2.9, indicating health care costs about three times that of the average Medicare beneficiary.

Patients also had a significant number of comorbid conditions associated with the logistic EuroSCORE covariates, including unstable angina $(30.7 \%)$, previous cardiac surgery (20.6\%), and recent myocardial infarction (17.6\%). Additionally, patients had clinically meaningful comorbid HCC conditions, including heart failure (71.4\%), cardiac arrhythmias $(71.0 \%)$, cardiorespiratory failure and shock $(33.7 \%)$, and vascular disease $(26.0 \%)$. Not surprisingly, statistically significant differences were found between the high-risk and non-high-risk groups for all logistic EuroSCORE covariates. Several additional comorbid conditions were statistically significant between the high-risk and non-high-risk groups, including certain manifestations of diabetes, renal failure and dialysis, ischemic stroke, and protein calorie malnutrition. 
Table I Baseline patient characteristics: surgically treated patients with severe aortic stenosis

\begin{tabular}{|c|c|c|c|c|}
\hline Characteristic & $\begin{array}{l}\text { All patients } \\
(n=1474)\end{array}$ & $\begin{array}{l}\text { HR } \\
(n=252)\end{array}$ & $\begin{array}{l}\text { NHR } \\
(n=1222)\end{array}$ & $P$ value \\
\hline Age (mean, SD, median)* & 74.0 & 77.6 & 73.3 & $<0.0001$ \\
\hline Female $(\%)$ & $40.7 \%$ & $52.8 \%$ & $38.2 \%$ & $<0.0001$ \\
\hline White $(\%)$ & $93.1 \%$ & $93.7 \%$ & $93.0 \%$ & 0.729 \\
\hline East region (CMS regions $\mathrm{I}-4)$ & $51.1 \%$ & $51.6 \%$ & $51.0 \%$ & 0.861 \\
\hline Central region (CMS regions $5-7$ ) & $34.3 \%$ & $36.1 \%$ & $33.9 \%$ & 0.497 \\
\hline West region (CMS regions 8-10) & $14.7 \%$ & $12.3 \%$ & $15.1 \%$ & 0.246 \\
\hline \multicolumn{5}{|l|}{ Logistic EuroSCORE comorbidity components (\%) } \\
\hline Chronic pulmonary disease & $9.1 \%$ & $17.5 \%$ & $7.4 \%$ & $<0.000$ I \\
\hline Extracardiac arteriopathy & $14.9 \%$ & $31.3 \%$ & $11.5 \%$ & $<0.0001$ \\
\hline Neurologic dysfunction & $7.0 \%$ & $19.4 \%$ & $4.4 \%$ & $<0.0001$ \\
\hline Previous cardiac surgery & $20.6 \%$ & $63.1 \%$ & $11.8 \%$ & $<0.0001$ \\
\hline Medicare ESRD beneficiary (serum creatinine $>200$ ) & $3.9 \%$ & $9.5 \%$ & $2.8 \%$ & $<0.0001$ \\
\hline Active endocarditis & $5.0 \%$ & $13.5 \%$ & $3.2 \%$ & $<0.0001$ \\
\hline Critical preoperative state & $17.6 \%$ & $51.2 \%$ & $10.7 \%$ & $<0.0001$ \\
\hline Unstable angina & $30.7 \%$ & $55.2 \%$ & $25.6 \%$ & $<0.0001$ \\
\hline Left ventricular dysfunction & $5.8 \%$ & $13.1 \%$ & $4.3 \%$ & $<0.0001$ \\
\hline Recent myocardial infarction & $17.6 \%$ & $36.9 \%$ & $13.6 \%$ & $<0.0001$ \\
\hline Pulmonary hypertension & $10.2 \%$ & $21.8 \%$ & $7.8 \%$ & $<0.0001$ \\
\hline Logistic EuroSCORE (mean, SD, median) & $12 \%$ & $37 \%$ & $7 \%$ & $<0.0001$ \\
\hline Logistic EuroSCORE $\geq 0.20(\%)$ & $17.1 \%$ & $100.0 \%$ & $0.0 \%$ & NA \\
\hline \multicolumn{5}{|c|}{ Comorbid conditions not already included in logistic EuroSCORE (\%) (Medicare HCC group) } \\
\hline HCC7 - Metastatic cancer and acute leukemia & $1.4 \%$ & $* *$ & ** & 0.811 \\
\hline HCC8 - Lung, upper digestive tract, and other severe cancers & $1.1 \%$ & ** & ** & 0.623 \\
\hline HCC9 - Lymphatic, head and neck, brain, and other major cancers & $3.1 \%$ & ** & ** & 0.780 \\
\hline $\mathrm{HCClO}-$ Breast, prostate, colorectal and other cancers and tumors & $13.2 \%$ & $13.5 \%$ & $13.1 \%$ & 0.865 \\
\hline $\mathrm{HCCI} 5$ - Diabetes with renal or peripheral circulatory manifestation & $7.1 \%$ & $15.5 \%$ & $5.3 \%$ & $<0.0001$ \\
\hline $\mathrm{HCCl} 6$ - Diabetes with neurologic or other specified manifestation & $5.6 \%$ & $5.6 \%$ & $5.6 \%$ & 0.995 \\
\hline HCCI8 - Diabetes with ophthalmologic or unspecified manifestation & $2.8 \%$ & $* *$ & $* *$ & 0.997 \\
\hline $\mathrm{HCCl} 9$ - Diabetes without complication & $23.3 \%$ & $26.6 \%$ & $22.7 \%$ & 0.180 \\
\hline HCC2I - Protein-calorie malnutrition & $6.7 \%$ & $13.9 \%$ & $5.2 \%$ & $<0.0001$ \\
\hline HCC38 - Rheumatoid arthritis and inflammatory connective tissue disease & $7.5 \%$ & $6.0 \%$ & $7.9 \%$ & 0.297 \\
\hline HCC55 - Major depressive, bipolar, and paranoid disorders & $4.1 \%$ & $* *$ & ** & 0.882 \\
\hline HCC79 - Cardiorespiratory failure and shock & $33.7 \%$ & $51.6 \%$ & $30.0 \%$ & $<0.0001$ \\
\hline HCC 80 - Congestive heart failure & $71.4 \%$ & $88.5 \%$ & $67.9 \%$ & $<0.0001$ \\
\hline HCC8I - Acute myocardial infarction & ** & $* *$ & ** & 0.521 \\
\hline HCC82 - Unstable angina and other acute ischemic heart disease & $5.9 \%$ & $8.3 \%$ & $5.4 \%$ & 0.072 \\
\hline HCC92 - Specified heart arrhythmias & $71.0 \%$ & $81.7 \%$ & $68.7 \%$ & $<0.0001$ \\
\hline HCC96 - Ischemic or unspecified stroke & $11.3 \%$ & $16.7 \%$ & $10.2 \%$ & 0.0003 \\
\hline HCCIO0 - Hemiplegia/hemiparesis & $2.2 \%$ & $4.4 \%$ & $1.8 \%$ & 0.012 \\
\hline HCCIO4 - Vascular disease with complications & $10.2 \%$ & $17.1 \%$ & $8.8 \%$ & $<0.0001$ \\
\hline HCCI05 - Vascular disease & $26.0 \%$ & $31.7 \%$ & $24.8 \%$ & 0.022 \\
\hline HCCI08 - Chronic obstructive pulmonary disease & $5.2 \%$ & $7.5 \%$ & $4.7 \%$ & 0.060 \\
\hline $\mathrm{HCCI} 19$ - Proliferative diabetic retinopathy and vitreous hemorrhage & $1.5 \%$ & ** & $* *$ & 0.003 \\
\hline $\mathrm{HCCl} 30$ - Dialysis status & $3.6 \%$ & $9.1 \%$ & $2.5 \%$ & $<0.0001$ \\
\hline $\mathrm{HCC}|3|$ - Renal failure & $8.3 \%$ & $15.9 \%$ & $6.7 \%$ & 0.0001 \\
\hline HCC score (mean, SD, median) & 2.85 & 4.14 & 2.58 & $<0.0001$ \\
\hline
\end{tabular}

Notes: *Part of the Logistic EuroSCORE; ** $\leq 10$ patients.

Abbreviations: CMS, Centers for Medicare and Medicaid Services; ESRD, end-stage renal disease; HCC, Hierarchical Condition Category; SD, standard deviation; HR, high-risk; NHR, non-high-risk; EuroSCORE, European System for Cardiac Operative Risk Evaluation.

\section{Clinical outcomes}

Results of the unadjusted clinical outcomes analysis indicated that almost $40 \%$ of patients had died by the end of the study period, with $61.1 \%$ of patients still alive at the end of 5 years (Figure 1). High-risk patients had significantly lower survival rates than non-high-risk patients $(P<0.0001)$, with rates of $33.3 \%$ and $66.8 \%$ at 5 years, respectively. Mean follow-up time across all patients was 3.8 years, while the high-risk group was 2.7 and the non-high-risk group was $4.0(P \leq 0.0001)$. 


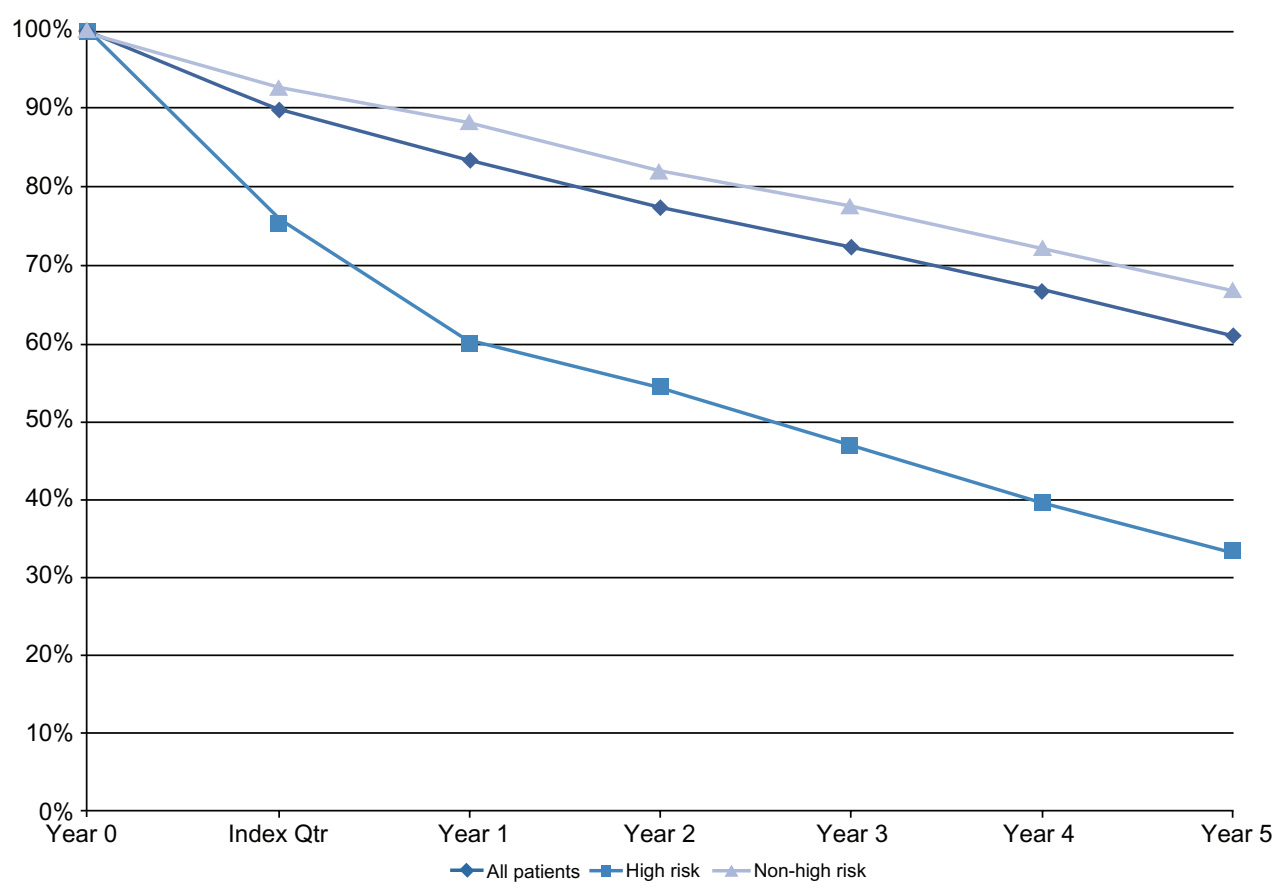

Figure I Survival rates over 5 years for the overall population (all patients), high-risk patients, and non-high-risk patients. Note: Percentages indicate the proportion of patients alive at the end of each year.

Additionally, a larger proportion of high-risk patients also experienced myocardial infarctions $(4.8 \%$ versus $2.0 \%$, $P=0.009)$ and strokes $(7.5 \%$ versus $6.6 \%, P=0.601)$ during the 5-year follow-up period (Table 2).

Significant predictors of mortality included patient age, three separate logistic EuroSCORE components (neurologic dysfunction, Medicare ESRD status, critical preoperative state), and five comorbid conditions, even after adjusting for confounding factors (Table 3 ). The risk stratification variable defined by a logistic EuroSCORE $>20 \%$, was not included in the final model because the individual logistic EuroSCORE components were used in the model. Medicare ESRD beneficiaries were found to have a more than three-fold increase in risk of death (95\% CI 1.97-6.65, $P<0.0001)$. Similarly, patients in a critical perioperative state and those suffering from metastatic cancer and acute leukemia both demonstrated significant increases in risk of death of more than 2.5 times (95\% CI 2.07-3.08 and 1.54-4.31, respectively).

\section{Resource use and costs}

An analysis of health care resource use (Table 4) over the 5 -year period revealed an average of 4.0 acute inpatient hospitalizations (3.0 excluding index hospitalization) per patient, with high-risk and non-high-risk patients incurring 4.7 and 3.9 admissions, respectively $(P=0.003,3.7$ and 2.9 admissions excluding index event). On average, patients were hospitalized for 32.0 days, with high-risk patients incurring 46.6 days and non-high-risk patients incurring 29.0 days $(P \leq 0.0001)$. Overall, $1.4 \%$ of patients underwent a repeat AVR procedure, with the high-risk patients undergoing the procedure more frequently. Over half (64.3\%) of all patients received home health care, while more than a third receiving skilled nursing facility care (37.1\%). Approximately $68 \%$ of all patients needed some type of durable medical equipment to manage their care. Univariate results showed statistically significant differences between high-risk and non-high-risk patients for hospital admissions and hospital days, skilled

Table 2 Clinical outcomes at 5 years

\begin{tabular}{lllll}
\hline Clinical outcome & $\begin{array}{l}\text { 5-year rate } \\
\text { All patients }\end{array}$ & HR & NHR & P value \\
\hline Survival (n, \% of patients) & $900,61.1 \%$ & $84,33.3 \%$ & $816,66.8 \%$ & $<0.0001$ \\
Follow-up years (mean) & 3.8 & 2.7 & 4.0 & $<0.0001$ \\
Myocardial infarction (n, \% of patients) & $36,2.4 \%$ & $12,4.8 \%$ & $24,2.0 \%$ & 0.009 \\
Stroke (n, \% of patients) & $100,6.8 \%$ & $19,7.5 \%$ & $81,6.6 \%$ & 0.601 \\
\hline
\end{tabular}

Abbreviations: HR, high-risk; NHR, non-high-risk. 
Table 3 Significant predictors of 5-year mortality

\begin{tabular}{|c|c|c|c|}
\hline Covariate & Hazards ratio & $95 \% \mathrm{Cl}$ & $P$ \\
\hline \multicolumn{4}{|l|}{ Patient demographics } \\
\hline Age & 1.023 & $1.012-1.035$ & $<0.0001$ \\
\hline \multicolumn{4}{|l|}{ Logistic EuroSCORE components } \\
\hline Neurologic dysfunction & 1.729 & $|.293-2.31|$ & 0.0002 \\
\hline Medicare ESRD beneficiary & 3.619 & $1.969-6.653$ & $<0.0001$ \\
\hline Critical preoperative state & 2.528 & $2.073-3.083$ & $<0.0001$ \\
\hline \multicolumn{4}{|l|}{ Comorbid conditions not already included in logistic EuroSCORE } \\
\hline HCC7 - Metastatic cancer and acute leukemia & 2.579 & $1.542-4.315$ & 0.0003 \\
\hline HCCIO - Breast, prostate, colorectal and other cancers and tumors & 1.369 & $1.079-1.738$ & 0.010 \\
\hline HCC2I - Protein-calorie malnutrition & 1.821 & $1.390-2.387$ & $<0.0001$ \\
\hline HCC79 - Cardiorespiratory failure and shock & 1.650 & $1.383-1.969$ & $<0.0001$ \\
\hline HCC80 - Congestive heart failure & 1.549 & $1.220-1.966$ & 0.0003 \\
\hline
\end{tabular}

Abbreviations: ESRD, end-stage renal disease; Cl, confidence interval; HCC, Hierarchical Condition Category; EuroSCORE, European System for Cardiac Operative Risk Evaluation.

nursing facility admissions $(49.6 \%, 34.5 \%, P \leq 0.0001)$, skilled nursing facility days $(27.8,16.3, P=0.001)$, admissions to hospice care $(12.3 \%, 7.4 \%, P=0.009)$, admissions to long-term care hospitals $(9.9 \%, 4.7 \%, P=0.001)$, dialysis services $(9.5 \%, 3.6 \%, P \leq 0.0001)$, and outpatient hospital care $(83.7 \%, 95.7 \%, P \leq 0.0001)$.

Total health care costs (including index quarter costs) over the 5-year period were $\$ 112,758$ per patient on average (Figure 2). The majority of costs were for acute inpatient hospitalizations $(\$ 69,338[61.5 \%])$, physician services (\$19,644 [17.4\%]), skilled nursing care (\$5163 [4.6\%]), and home health care (\$4399 [3.9\%]). The remaining costs were fairly evenly distributed among other types of health care services. Total 5-year costs were significantly different between the high-risk and non-high-risk groups $(\$ 144,183$ versus $\$ 106,277$ per patient, $P \leq 0.0001$ ). High-risk patients were $40 \%$ more costly than non-high-risk patients when inpatient hospital services were provided ( $\$ 91,771$ versus $\$ 64,712, P \leq 0.0001)$. Average skilled nursing ( $\$ 8291$ versus $\$ 4518, P \leq 0.0001$ ), home health ( $\$ 6642$ versus $\$ 3937, P=0.0146)$, and long-term hospital care ( $\$ 4139$ versus $\$ 1564, P=0.0006$ ) were all more costly for highrisk patients.

Total follow-up costs alone (excluding index quarter costs) over the 5-year period were $\$ 59,855$. High-risk patients had $55 \%$ higher follow-up costs than non-high-risk patients $(\$ 85,731$ versus $\$ 55,456)$. Hospital inpatient follow-up costs represented the largest proportion of total follow-up costs within each group, with $41.4 \%$ and $38.8 \%$ in the high-risk and non-high-risk groups, respectively. Inpatient follow-up costs for high-risk patients were $\$ 35,493$, whereas the average inpatient hospitalization cost for non-high-risk

Table 4 Five-year health resource use

\begin{tabular}{|c|c|c|c|c|}
\hline Health care resource & All patients & HR & NHR & $P$ value \\
\hline Acute inpatient ( $\mathrm{n}, \%$ patients) & $1474,100 \%$ & $252,100 \%$ & $1,222,100 \%$ & NA \\
\hline Hospitalizations (mean, SD)* & 4.0 & 4.7 & 3.9 & 0.003 \\
\hline Hospital days (mean, SD)* & 32.0 & 46.6 & 29.0 & $<0.0001$ \\
\hline Repeat AVR ( $\mathrm{n}, \%$ patients) & $20,1.4 \%$ & $* *$ & $* *$ & 0.728 \\
\hline Valvuloplasty (n, \% patients) & $* *$ & $* *$ & $* *$ & 0.6496 \\
\hline Long-term care hospital (n, \% patients) & $83,5.6 \%$ & $25,9.9 \%$ & $58,4.7 \%$ & 0.001 \\
\hline Inpatient rehabilitation facility ( $\mathrm{n}, \%$ patients) & $300,20.4 \%$ & $56,22.2 \%$ & $244,20.0 \%$ & 0.418 \\
\hline Skilled nursing facility (n, \% patients) & $547,37.1 \%$ & $125,49.6 \%$ & $422,34.5 \%$ & $<0.0001$ \\
\hline Number of SNF days (mean, SD) & 18.3 & 27.8 & 16.3 & 0.001 \\
\hline Hospice care (n, \% patients) & $|2|, 8.2 \%$ & $31,12.3 \%$ & $90,7.4 \%$ & 0.009 \\
\hline Home health care (n, \% patients) & $948,64.3 \%$ & $156,61.9 \%$ & $792,64.8 \%$ & 0.380 \\
\hline Outpatient hospital care (n, \% patients) & $138 \mid, 93.7 \%$ & $211,83.7 \%$ & $1170,95.7 \%$ & $<0.0001$ \\
\hline Physician/supplier services (n, \% patients) & $1474,100 \%$ & $252,100 \%$ & $1221,100 \%$ & NA \\
\hline Durable medical equipment use ( $\mathrm{n}, \%$ patients) & $1000,67.8 \%$ & $170,67.5 \%$ & $830,67.9 \%$ & 0.887 \\
\hline Dialysis services ( $n, \%$ patients) & $68,4.6 \%$ & $24,9.5 \%$ & $44,3.6 \%$ & $<0.0001$ \\
\hline
\end{tabular}

Notes: *Includes index hospitalization; $* * \leq 10$ patients.

Abbreviations: AVR, aortic valve replacement; HR, high-risk; NHR, non-high-risk; SD, standard deviation; SNF, skilled nursing facility. 


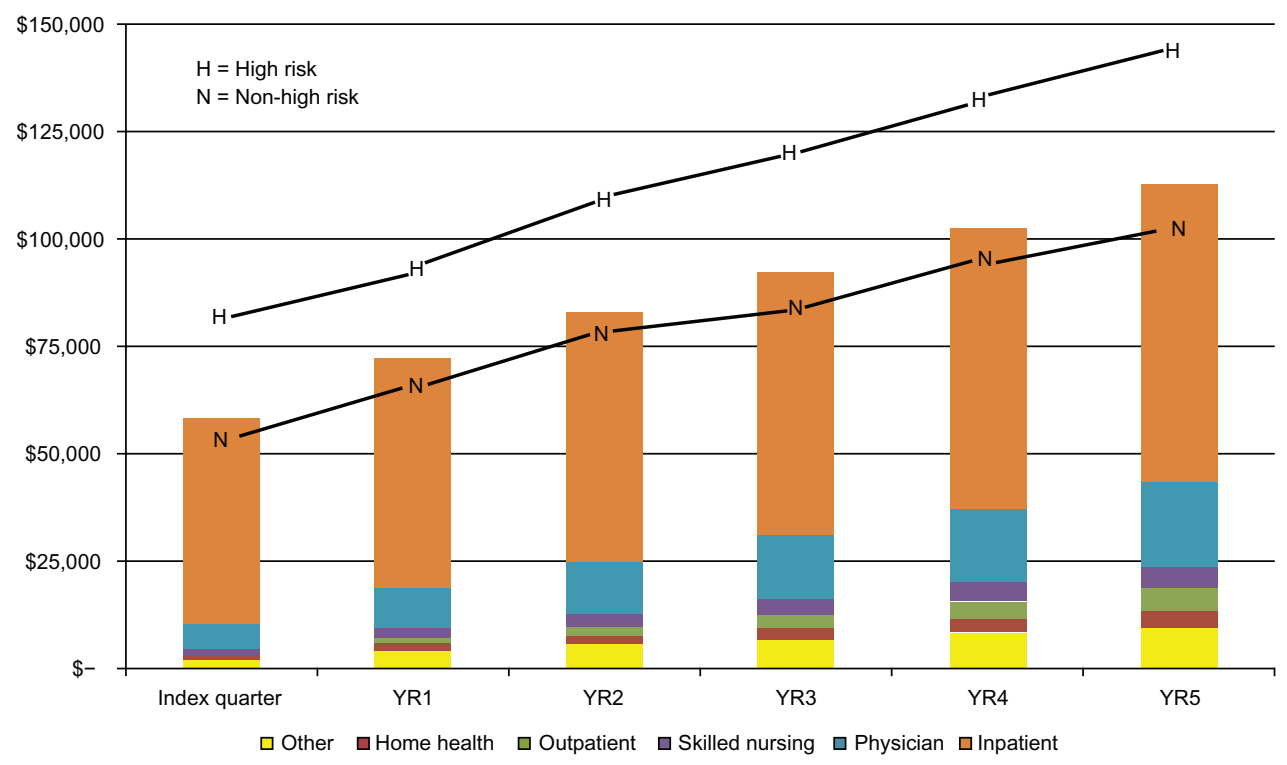

Figure 2 Average 5-year cumulative costs per patient.

Note: Mean cumulative costs per patient over a 5-year period, with total high-risk and non-high-risk costs over time highlighted.

patients during the follow-up period was $\$ 21,517$. Limited survival at the end of the follow-up period may have decreased costs to a lower than expected level. Therefore, we also examined the average annual follow-up costs (total costs minus index hospitalization costs) per patient by dividing the patient's total follow-up costs by the number of follow-up years. The overall average follow-up costs per patient per year was $\$ 15,244$, with high-risk patients being twice as costly per year as non-high-risk patients $(\$ 26,891$ versus $\$ 13,686)$.

\section{Discussion}

In a Medicare sample of 1474 AVR patients, $17.1 \%$ had a EuroSCORE $\geq 20 \%$ and were considered high-risk. Not surprisingly, the high-risk group had worse outcomes and higher resource use and costs than the non-high-risk group. High-risk patients had significantly lower survival (33.3\% versus $66.8 \%)$, higher rates of myocardial infarction $(4.8 \%$ versus $2.0 \%$ ) and stroke (7.5\% versus $6.6 \%$ ), more follow-up hospitalizations (3.7 versus 2.9 ), and required more postacute care services in general. Total health care costs over the 5-year period reached $\$ 112,758$ per patient overall, with the high-risk group driving costs higher $(\$ 144,183$ versus $\$ 106,277$ in the non-high-risk group). These results are consistent with our hypothesis that high-risk AVR patients are more costly and have worse outcomes than non-highrisk patients.

Recent results from PARTNER (Placement of AoRTic TraNscathetER Valve Trial) of high-risk patients undergoing transcatheter AVR are comparable with the results presented here. The PARTNER Cohort A study compared high-risk patients randomized to either transcatheter AVR or standard AVR, while the Cohort B study examined "inoperable" high-risk patients randomized to transcatheter AVR or standard medical care. Patients in the surgical AVR arm of the "operable" cohort had an average one-year survival rate of $73.2 \%$, while those in the "inoperable" transcatheter AVR arm had a one-year survival rate of $69.3 \% .^{14,15}$ The oneyear survival rate found in the high-risk Medicare population in this study was $66.8 \%$. Although somewhat lower, it should be noted that the average logistic EuroSCORE in our high-risk group was $36.8 \%$, while operable AVR and inoperable transcatheter AVR patients in PARTNER had logistic EuroSCOREs of $29.2 \%$ and $26.4 \%$, respectively.

In terms of resource use and costs, only results from the inoperable transcatheter AVR patient group have been published and can be compared with the high-risk patients in this study. The high-risk AVR population in this study incurred 3.7 subsequent hospitalizations over the 5-year follow-up period (with 1.4 total and 0.5 cardiovascularrelated hospitalizations in the first year). The inoperable transcatheter AVR population incurred 1.02 total follow-up hospitalizations and 0.50 cardiovascular hospitalizations during the first year. ${ }^{18}$ One-year costs for high-risk Medicare AVR patients in 2003 were $\$ 96,476$. This compares with a total of $\$ 107,892$ in one-year costs for the high-risk inoperable transcatheter AVR population. ${ }^{18}$ The results found in this Medicare claims analysis compare well with high-risk transcatheter AVR patients. We await additional results from 
the surgical AVR arm of the PARTNER trial for additional comparisons.

The incidence of severe, symptomatic aortic stenosis is expected to increase with the aging of the population. Previous studies have demonstrated that outcomes are poor in untreated patients and that the only effective treatment is AVR. ${ }^{2-13}$ Although our results demonstrating higher costs and worse outcomes for high-risk versus non-high-risk AVR patients may not be surprising, there have been no studies to date examining the health care costs and resource use in this patient population (the PARTNER trial focused on high-risk patients only.) Additionally, studies of other procedures only compare immediate surgical outcomes of high-risk and non-high risk patients. More limited data exist that compare long-term outcomes and especially costs for high-risk versus non-high risk patients. One example of benchmark data in this area is for abdominal aortic aneurysm repair. Steinmetz et al examined high-risk surgical and endovascular abdominal aneurysm repair compared with low-risk surgical abdominal aneurysm repair outcomes over 7 years. ${ }^{19}$ However, no cost or resource use data were reported.

As with any study that uses administrative claims data to track outcomes and costs, there are limitations. First, administrative claims data were not originally designed to answer research questions; they exist as a byproduct of the health insurance claims payment process. Clinical conditions and procedures are identified in claims data using various diagnosis and procedure coding systems. Often times, the codes are ambiguous and do not allow enough specificity for tracking specific outcomes and risk factors of interest. The "costs" provided in the dataset must be defined as "payments" made by payers (or, alternatively, billed charges). The Medicare data probably specifically underestimate total costs due to incomplete Medicare-covered benefits, such as prescription drugs and the majority of skilled nursing services.

In spite of these limitations, claims data are often used for this type of longitudinal research. Additionally, claims data provide insight into outcomes of patient populations that are not required to meet the restrictive enrollment criteria of clinical trials and may be more representative of how patients are truly treated. The Medicare database in particular is one of the most comprehensive datasets available to study costs and outcomes in an elderly population. Because the majority of patients with aortic stenosis tend to be elderly, these data are ideal for understanding this group.

In our sample, a total of 1474 high-risk and non-highrisk Medicare patients underwent AVR during 2003. Because our data represent a $5 \%$ random sample of all
Medicare beneficiaries during that year, we can project that approximately 30,000 Medicare patients had surgical AVR in 2003. Total costs for the patient population over the 5-year follow-up period can be estimated at $\$ 3.4$ billion $(30,000 \times \$ 112,758)$. Additionally, we found important differences in costs, health resource use, and outcomes between high-risk and non-high-risk patients, with high-risk patients incurring higher costs, more health resource use, and greater mortality. Importantly, even though high-risk patients tended to have higher mortality (thus, fewer years of follow-up), the average total follow-up costs per high-risk patient was $55 \%$ higher at $\$ 85,731$ versus $\$ 55,456$. These findings underscore the need for future studies comparing the outcomes and health care costs of high-risk patients treated with surgical AVR compared with those treated with transcatheter AVR to determine whether transcatheter AVR provides a lower cost but clinically beneficial solution to the management of these high-risk patients. This study provides detailed cost and health resource use data on both high-risk and non-high-risk surgical AVR patients, which is helpful in providing comparative cost benchmarks for new, minimally invasive approaches.

\section{Disclosure}

AKT and FGD are employees of Edwards Lifesciences. MAC, BS, and MK are consultants on a project funded by Edwards Lifesciences. DC receives research support from Edwards Lifesciences and Medtronic. ROB has a consulting relationship with Edwards Lifesciences. LGS serves as a clinical investigator in studies funded by industry but has no direct financial relationship with industry to disclose.

\section{References}

1. HCUPnet. Healthcare Cost and Utilization Project. Rockville, MD: Agency for Healthcare Research and Quality; 1993-2010. Available from: http://hcupnet.ahrq.gov/. Accessed July 9, 2012.

2. Varadarajan P, Kapoor N, Bansal R, Pai R. Survival in elderly patients with severe aortic stenosis is dramatically improved by aortic valve replacement: results from a cohort of 277 patients aged $\geq 80$ years. Eur J Cardiothorac Surg. 2006;30:722-727.

3. Kang DH, Park SJ, Rim JH, et al. Early surgery versus conventional treatment in asymptomatic very severe aortic stenosis. Circulation. 2010;121:1502-1509.

4. Bouma BJ, Van den Brink RBA, Van der Meulen JHP, et al. To operate or not on elderly patients with aortic stenosis: the decision and its consequences. Heart. 1999;82:143-148.

5. Bach DS, Siao D, Girard SE, Duvernoy EC, McCallister BD Jr, Gualano SK. Evaluation of patients with severe symptomatic aortic stenosis who do not undergo aortic valve replacement: the potential role of subjectively overestimated operative risk. Circ Cardiovasc Qual Outcomes. 2009;2:533-539.

6. Sundt TM, Bailey MS, Moon MR, et al. Quality of life after aortic valve replacement at the age of $>80$ years. Circulation. 2000;102: III70-III74. 
7. Smith N, McAnulty JH, Rahimtoola SH. Severe aortic stenosis with impaired left ventricular function and clinical heart failure: results of valve replacement. Circulation. 1978;58:255-264.

8. Murphy ES, Lawson RM, Starr A, Rahimtoola SH. Severe aortic stenosis in patients 60 years of age or older: left ventricular function and 10-year survival after valve replacement. Circulation. 1981;64:II184-II188.

9. Lund O. Preoperative risk evaluation and stratification of long-term survival after valve replacement for aortic stenosis: reasons for earlier operative intervention. Circulation. 1990;82:124-139.

10. Kouchoukos NT, Davila-Roman VG, Spray TL, Murphy SF, Perrillo JB. Replacement of the aortic root with a pulmonary autograft in children and young adults with aortic-valve disease. $N$ Engl J Med. 1994;330:1-6.

11. Connolly HM, Oh JK, Orszulak TA, et al. Aortic valve replacement for aortic stenosis with severe left ventricular dysfunction: prognostic indicators. Circulation. 1997;95:2395-2400.

12. Kvidal P, Bergstrom R, Horte LG, Stahle E. Observed and relative survival after aortic valve replacement. J Am Coll Cardiol. 2000;35: 747-756.

13. Schwarz F, Baumann P, Manthey J, et al. The effect of aortic valve replacement on survival. Circulation. 1982;66:1105-1110.
14. Leon M, Smith C, Mack M, et al. Transcatheter aortic-valve implantation for aortic stenosis in patients who cannot undergo surgery. $N$ Engl J Med. 2010;353:1597-1607.

15. Smith CR, Leon MB, Mack MJ, et al. Transcatheter versus surgical aortic-valve replacement in high-risk patients. $N$ Engl $\mathrm{J} \mathrm{Med}$. 2011;364:2187-2198.

16. Florath I, Albert A, Boening A, Ennker IC, Ennker J. Aortic valve replacement in octogenarians: identification of high-risk patients. Eur J Cardiothorac Surg. 2010;37:1304-1310.

17. Pope GC, Kautter J, Ellis R, et al. Risk adjustment of Medicare capitation payments using the CMS-HCC model. Health Care Financ Rev. 2004;25:119-141.

18. Reynolds MR, Magnuson EA, Wang K, et al. Cost effectiveness of transcatheter aortic valve replacement compared with standard care among inoperable patients with severe aortic stenosis: Results from The PARTNER Trial (Cohort B). Circulation. 2012;125:1102-1109.

19. Steinmetz E, Abello N, Kretz B, Gauthier E, Bouchot O, Brenot R. Analysis of outcome after using high-risk criteria selection to surgery versus endovascular repair in the modern era of abdominal aortic aneurysm treatment. Eur J Vasc Endovasc Surg. 2010;39:403-409. 


\section{Appendix}

Appendix A Logistic EuroSCORE definitions

\begin{tabular}{|c|c|}
\hline Risk factors & Definition \\
\hline \multicolumn{2}{|l|}{ Patient-related factors } \\
\hline Age & Age in years at the beginning of 2003 \\
\hline Gender & Female \\
\hline Chronic pulmonary disease & $\begin{array}{l}\text { At least one hospitalization or outpatient hospital visit with a principal diagnosis } 4910,49120,49121,49122 \text {, } \\
4918,4919,4920,4928,4940,4941,496,514,515,51883,51884,51889,5199\end{array}$ \\
\hline Extracardiac arteriopathy & $\begin{array}{l}\text { At least one hospitalization or outpatient hospital visit with a principal diagnosis, 4I 78, 433I0, 433II, 43320, } \\
4332 \text { I, 43330, 4333I, 43380, 4338I, 43390, 4339I, 43490, 4349I, 435I, 4353, 4358, 4359, 4370, 44020, 4402I, } \\
\text { 44022, 44023, 44024, 44029, 44030, 4403I, 44032, 4404, 4408, 44389, 4439, 447I, 4479 }\end{array}$ \\
\hline Neurological dysfunction & 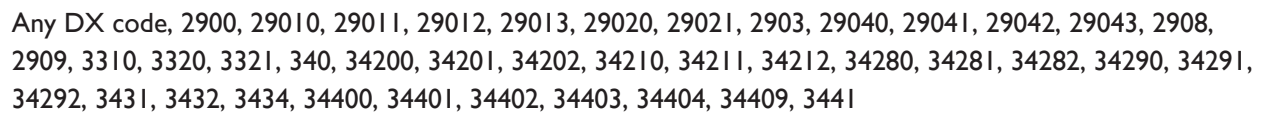 \\
\hline Previous cardiac surgery & 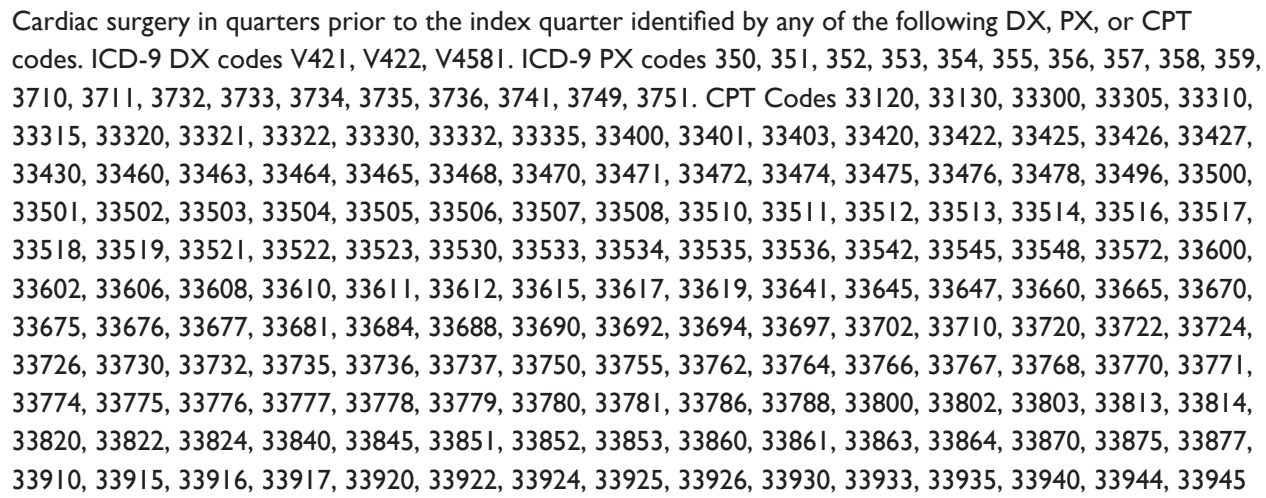 \\
\hline Serum creatinine & Patient is afflicted with ESRD and on dialysis during index year (ESRD indicator field in denominator file) \\
\hline Active endocarditis & Any of the following DX codes appearing in the same quarter of the index hospitalization $=4210,4211,4219$ \\
\hline Critical preoperative state & $\begin{array}{l}\text { Certain DX or PX on the same index hospitalization claim. DX 4274I, 4275, 5845, 5846, 5847, 5848, 5849, } \\
\text { VI } 253 \text {, V432I. PX 3752, 3753, 3754, 3755, 3760, 376I, 3762, 3763, 3764, 3765, 3766, 3767, } 3768 .\end{array}$ \\
\hline \multicolumn{2}{|l|}{ Cardiac-related factors } \\
\hline Unstable angina & Any DX code $4 I I I$ \\
\hline Left ventricular dysfunction & Any DX code $42820,42821,42822,42823$ \\
\hline Recent myocardial infarction & 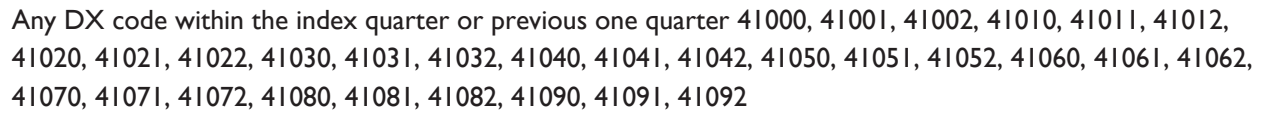 \\
\hline Pulmonary hypertension & Any DX code 4150 or 4160 \\
\hline \multicolumn{2}{|l|}{ Operation-related factors } \\
\hline Emergent operation & $\begin{array}{l}\text { Cardiac-related admission DX codes on patients who, on their AVR hospitalization claim, were admitted } \\
\text { through the ER upon recommendation of the ER physician }\end{array}$ \\
\hline Other than isolated CABG & NA \\
\hline Surgery on thoracic aorta & NA \\
\hline Postinfarct septal rupture & DX code 4297 I on same claim as any AVR hospitalization occurring during index quarter \\
\hline
\end{tabular}

Abbreviations: AVR, aortic valve replacement; ER, emergency room; CABG, coronary artery bypass grafting; ESRD, end-stage renal disease; DX, diagnosis; PX, procedure; CPT, Current Procedural Terminology; NA, not applicable.

\section{Publish your work in this journal}

Risk Management and Healthcare Policy is an international, peerreviewed, open access journal focusing on all aspects of public health, policy, and preventative measures to promote good health and improve morbidity and mortality in the population. The journal welcomes submitted papers covering original research, basic science, clinical \& epidemio- logical studies, reviews and evaluations, guidelines, expert opinion and commentary, case reports and extended reports. The manuscript management system is completely online and includes a very quick and fair peerreview system, which is all easy to use. Visit http://www.dovepress.com/ testimonials.php to read real quotes from published authors. 